\title{
HPV E6/E7-encoding Semliki Forest Virus Vaccine Vvax001
}

National Cancer Institute

\section{Source}

National Cancer Institute. HPV E6/E7-encoding Semliki Forest Virus Vaccine Vvax001. NCI

Thesaurus. Code C148539.

A vaccine consisting of a recombinant, attenuated, replication-incompetent form of the Semliki Forest Virus (SFV) vector encoding the viral oncoproteins E6 and E7 derived from the human papillomavirus (HPV), with potential immunomodulating and antineoplastic activities. Upon intramuscular administration, HPV E6/E7-encoding SFV vaccine Vvax001 induces expression of the E6/E7 proteins and stimulates both the innate and the adaptive immune system, resulting in a potent cytotoxic T-lymphocyte (CT L) response against and lysis of tumor cells expressing HPV E6 and E7. Oncoproteins E6 and E7 play a key role in the development of cervical intraepithelial neoplasia (CIN) and cervical carcinoma. 